The Open Materials Science Journal
CrossMark
Content list available at: www.benthamopen.com/TOMSJ/
DOI: $10.2174 / 1874088 \mathrm{X} 01610010018$

EDITORIAL

\title{
Advanced Microelectronic and Nanoscale Semiconductor Materials \& Applications
}

The continued shrinkage of micron-scale and nanoscale electronics and optoelectronics has required considerable challenges to be overcome in recent years. Novel and improved metrologies are required for supporting progress in the functionality, performance, cost-effectiveness, and mass production of devices and integrated components [1 - 12]. Therefore, additional processes and architectural and material requirements are necessary and are currently imposed for the advanced manufacturing of such devices. In this special issue, we address six parts (seven articles) indicating technological progress. We identified six key elements that a new fabrication technology must satisfy to overcome the challenges of ever-decreasing dimensions.

This special issue begins by reviewing seven articles regarding all aspects of characterization technology required for the development and manufacture of advanced microelectronic and optoelectronic devices. The majority of this special issue reviews papers covering essential concerns in optoelectronics, communication components, taste sensors, electromagnetic shielding materials, nanocontact printing, cascode low noise amplifiers (LNAs), and associative memory applications.

Part 1 describes a paper reporting the thermal annealing influence on the emission characteristic of n-gallium nitride $(\mathrm{GaN})$ materials and $\mathrm{GaN}$ devices. In addition, the luminescence intensity and emission life time were improved at a typical annealing temperature.

Part 2 describes a study that fabricated gallium arsenide (GaAs) power metal-semiconductor field effect transistors (MESFETs) by using double ion implantation technology for wireless communications. The power performance of the MESFET for the code division multiple access (CDMA) wireless communication applications was studied.

Part 3 describes a study in which the authors developed a chemical sensor array with seven chemical sensors. The designed sensors were categorized into two groups: the first group mainly comprises conductive polymer, and the second group comprises of a biomimic material. Furthermore, six sports drinks were identified and classified in the salt area.

Part 4 focuses mainly on the characteristic analysis of various electromagnetic shielding materials by using the various shielding effectiveness (SE)-measuring methods developed by TDK and ASTM.

Part 5 describes a paper on nanocontact printing technology, which is a promising next-generation lithography technique because of its low-cost and high-throughput patterning. Nanocontact printing by using hydrogen silsesquioxane soft stamps was studied for fabricating nanobio devices.

Finally, Part 6 describes a study in which two electronic applications, cascode LNA and associative memory, were implemented in a complementary metal-oxide semiconductor (CMOS) circuit involving submicron silicon technologies.

\section{REFERENCES}

[1] Amir Parviz B, Ryan D, Whitesides GM. Using self-assembly for the fabrication of nano-scale electronic and photonic devices. IEEE Trans Adv Packag 2003; 26(3): 233-41. [http://dx.doi.org/10.1109/TADVP.2003.817971]

[2] Chang GK, Guidotti D, Liu F, et al. Chip-to-chip optoelectronics SOP on organic boards or packages. IEEE Trans Adv Packag 2004; 27(2): 386-97. 
[http://dx.doi.org/10.1109/TADVP.2004.831880]

[3] Tsukada M, Tagami K, Hirose K, Kobayashi N. Theoretical bases of nano-scale devices. Proc $2^{\text {nd }}$ Int Con Adv Optoelectron Lasers; 2005; 2: $8-14$.

[4] Fok LM, Liu YH, Li WJ. Assembly of nano optics by an integrated probe-based system. $7^{\text {th }}$ IEEE Conference on Nanotechnology; 2007 Aug 2-5; HongKong: IEEE 2007; pp. 524-9. [http://dx.doi.org/10.1109/NANO.2007.4601246]

[5] Ahmed I, Er-Ping L. Time domain modeling: From nano-electronics to nano-photonics. Asia-Pacific Symposium on Electromagnetic Compatibility (APEMC); 2012 May 21-24; Singapore: IEEE, 1992; pp. 169-72. [http://dx.doi.org/10.1109/APEMC.2012.6237988]

[6] Joga R. Quantum dot floating gate transistor with multi-wall carbon nano tube channel for non-volatile memory devices. International Conference on Communication Systems and Network Technologies (CSNT); 2012 May 11-13; Rajkot, India: IEEE, 2012; pp. 774-9. [http://dx.doi.org/10.1109/CSNT.2012.169]

[7] Nour M, Ghoneim M, Droopad R, Hussain MM. CMOS compatible route for GaAs based large scale flexible and transparent electronics. 14 IEEE International Conference On Nanotechnology; 2014 Aug 18-24; Toronto, ON:IEEE 2014; pp. 835-8 [http://dx.doi.org/10.1109/NANO.2014.6968018]

[8] Hu Y, An B, Niu C, Lv W, Wu Y. Application of nano copper conductive ink for printed electronics. $15^{\text {th }}$ International Conference on Electronic Packaging Technology (ICEPT); 2014 Aug 12-15; Chengdu, India: IEEE 2014; pp. 1565-7. [http://dx.doi.org/10.1109/ICEPT.2014.6922953]

[9] Marshall A. Thoughts on possible future charge-based technologies for nano-electronics. IEEE Trans Circuits Syst 2014; $61(11)$ : $3057-65$. [http://dx.doi.org/10.1109/TCSI.2014.2335011]

[10] Trellenkamp S, Mikulics M, Winden A, et al. III-nitride nano-LEDs for single photon lithography. $10^{\text {th }}$ International Conference on Advanced Semiconductor Devices \& Microsystems (ASDAM); Germany: IEEE 2014; pp. 1-4.

[11] Collard D. Nano systems and nano scaled devices for new applications in biology and nanotechnology. International EUROSOI Workshop and International Conference on Ultimate Integration on Silicon (EUROSOI-ULIS); Bolonga, Italy: IEEE 2015; pp. 1-4. [http://dx.doi.org/10.1109/ULIS.2015.7063758]

[12] Hamdioui S. Electronics and computing in nano-era: The good, the bad and the challenging. $10^{\text {th }}$ International Conference on Design \& Technology of Integrated Systems in Nanoscale Era (DTIS); Naples, Italy: IEEE 2015; p. 1.

[http://dx.doi.org/10.1109/DTIS.2015.7127342]

Shen-Li Chen

Department of Electronic Engineering

National United University

MiaoLi City

Taiwan

Email: jackchen@nuu.edu.tw

(C) Shen-Li Chen; Licensee Bentham Open.

This is an open access article licensed under the terms of the Creative Commons Attribution-Non-Commercial 4.0 International Public License (CC BY-NC 4.0) (https://creativecommons.org/licenses/by-nc/4.0/legalcode), which permits unrestricted, non-commercial use, distribution and reproduction in any medium, provided the work is properly cited. 